\section{Liquor-CD8-T-(Suppressor)- Lymphozyten}

T. O. Kleine

Institut für Laboratoriumsmedizin und Pathobiochemie, Molekulare Diagnostik Standort Marburg Referenzlabor für Liquordiagnostik, UKGM Universitätsklinikum Gießen und Marburg, Marburg, Deutschland

Synonym(e) $\mathrm{CD}^{+} 8^{+}$Zellen im Liquor cerebrospinalis (CSF); Suppressor-/zytotoxische T-Zellen
Englischer Begriff CSF CD8 T lymphocytes; suppressor/ cytotoxic lymphocytes in CSF

Definition $\mathrm{CSF} \mathrm{CD}^{+} 8^{+}$-Zellen sind T-Zellen, die im Rahmen der Immunüberwachung in CSF bzw. Zentralnervensystem ihr passendes Antigenpeptid mithilfe von MHC-Klasse-I-Oberflächenmolekülen suchen $(\triangleright \mathrm{CD} 8)$. (s. a. $\triangleright$ Liquor cerebrospinalis) 